\title{
Comparación de pruebas de diagnóstico de mansonelosis en el estudio de prevalencia en comunidades del río Atabapo, Departamento del Guanía, Colombia 1996
}

\author{
Herbert I. Espitia ${ }^{1}$, Julián Trujillo ${ }^{2}$
}

\begin{abstract}
Resumen
En el departamento de Guainía, Colombia, se realizó un estudio tipo encuesta de prevalencia de corte en siete comunidades del río Atabapo, donde se calculó una muestra poblacional de 181 personas mayores de 10 años que se debían examinar, tomando, como unidad de análisis, 37 viviendas escogidas aleatoriamente. Se les tomó una muestra de sangre que se examinó mediante cuatro técnicas (examen en fresco, gota gruesa, prueba de Knott y conteo por $\mathrm{cm}^{3}$ ), examen médico, estudio de saneamiento y encuesta por vivienda. Se encontró una prevalencia de $57 \%$ en el grupo examinado que, por ser muestra representativa de la población, se puede decir que es la prevalencia de la zona. De las tres pruebas diagnósticas utilizadas, además de la prueba de Knott, el conteo por $\mathrm{cm}^{3}$ fue la que mejor resultado brindó en el estudio, dando una sensibilidad, especificidad y valor predictivo positivo similar y mejor que la de la prueba de oro que se tomó como referencia.
\end{abstract}

Comparison of mansonellosis diagnostic tests in a prevalency study in communities situated along the River Atabapo in the Guanía Department, Colombia, 1996

In the Guanía Department of Colombia a cross-sectional prevalence questionnaire was carried out in six communities along the River Atabapo, where a predetermined sample population of 181 people older than 10 years of age were examined, taking 37 previously selected dwellings as the cone analysis unit. All the inhabitants in the sample gave blood samples which were subjected to examination by means of four techniques (fresh blood sample, thick smear, Knott test and $\mathrm{cm}^{3}$ count), they had a medical examination, a sanitation study was made and a questionnaire was carried out in each dwelling. A 57\% prevalence was encountered in the sample population, thus one can say that it is the zone prevalence. Of the three diagnostic tests used in addition to the Knott test, $\mathrm{cm}^{3}$ count gave the study's best results, giving a sensitivity, specificity and positive predictive value similar to or better than the gold standard (the Knott test) which was taken as the point of reference.

La mansonelosis es un una enfermedad ocasionada por parásitos nemátodos no envainados llamados filarias que se distribuyen en amplias zonas del trópico y de regiones subtropicales.
El nemátodo con forma de filamento produce un variado grupo de lesiones en el ser humano que son características de acuerdo con la especie de filaria que lo infecte. Se transmiten a través

${ }^{1}$ Secretaría de Salud del Guainía

${ }^{2}$ Banco de Sangre, Hospital Inírida

Recibido para su publicación: febrero 10, 1998 
de la picadura de un diptero hemátofago como los Simulium, Culex, Mansonias, Tabanides y Culicoides (1-4). Las filariasis que presentan importancia médica son: Wuchereria bancrofti, Brugia malayi, Onchocerca volvulus, Loa loa, Ancathochelonema, Mansonella pertans y Mansonella ozzardi $(1,2)$. En el país se ha detectado mansonelosis en los departamentos de Casanare, Vichada, Meta, Vaupés y Guainía, en especial la producida por M. ozzardi $(5,6)$.

En el departamento del Guainía desde hace varios años se han diagnosticado microfilarias en sangre de personas que consultaron por presentar síntomas clínicos variados que inicialmente se relacionaron con paludismo y a quienes se les ordenaron exámenes de gota gruesa para el diagnóstico. Al realizar estos hallazgos en los resultados de la prueba de laboratorio estimularon la necesidad de realizar un estudio donde se pudiera establecer la prevalencia de la enfermedad en comunidades del Guainía.

Se escogieron las poblaciones que se encuentran a lo largo del río Atabapo para poder describir las características de la enfermedad, las variables relacionadas con la presencia de la mansonelas y evaluar las pruebas de laboratorio para su diagnóstico y establecer cuál puede ser la más sensible y específica.

\section{Materiales y métodos}

El departamento del Guainía se encuentra situado en el extremo centro-oriental de Colombia; tiene como límite al norte, el departamento del Vichada; al oriente, la república de Venezuela; al sur, la república de Brasil, y al occidente, los departamentos de Guaviare y Vaupés. El departamento tiene una extensión geográfica de $72.238 \mathrm{~km}^{2}$; su relieve es poco ondulado y presenta algunas elevaciones como la Serranía del Naquén, con una altura promedio de $200 \mathrm{~m}$ sobre el nivel del mar (7).

Posee una riqueza hidrográfica abundante; los principales ríos que surcan el territorio son el río Guaviare, el río Inírida, el río Guainía y el río Atabapo, además de los incontables caños y ríos menores que forman en conjunto una amplia red fluvial en toda la extensión del territorio del departamento, la cual es base del sistema de transporte y comunicaciones.
El clima del departamento es tropical cálido, con una temperatura media de $30^{\circ} \mathrm{C}$ y una humedad relativa de 70 a $90 \%$, con variaciones climáticas de los períodos de lluvia que conforman las dos estaciones, la de invierno (abril-noviembre) y la de verano (diciembre-marzo). La vegetación de la región está conformada en su gran mayoría por zonas de selva tropical húmeda, representada por bosques selváticos, y en una menor proporción presenta áreas de sabana, especialmente hacia el nororiente del departamento (7).

La zona escogida para el estudio comprendió las comunidades localizadas en la orilla del río Atabapo, frontera con Venezuela, 7 localidades que se encuentran entre la comunidad de Chaquita hasta la población de Raudal Pato, habitadas por indígenas de las etnia Curripaco, Baniva y Piapoco; el número de colonos en la zona es mínimo. La comunidad más cercana (Chaquita) se encuentra a 6 horas, aproximadamente, por vía fluvial desde el municipio de Inírida hasta unas 12 horas a la localidad más lejana (Raudal Pato). Esta es una zona de tránsito entre el municipio de Inírida y la parte media del río Guainía o río Negro; además, es área de colonización de grupos indígenas Curripacos procedentes del alto río Guainía.

Se realizó un estudio de encuesta de prevalencia, tipo corte, donde se tomó la vivienda como marco muestral, escogidas mediante muestreo aleatorio simple. Se realizó una historia clínica individual; se tomó una miestra de sangre a cada uno de los habitantes de las viviendas escogidas; se llevó a cabo una encuesta por vivienda sobre los aspectos básicos de la familia, ocupación y características de la habitación. También se llevo a cabo un estudio de saneamiento básico en cada comunidad estudiada. El número total de pacientes examinados se calculó con base en estudios previos realizados en el departamento del Guainía (5) y dio como resultado un total de 181.

Los exámenes de laboratorio se practicaron a cada uno de los habitantes de las viviendas escogidas y se les realizó el examen médico. Se les tomó una muestra sanguínea de $5 \mathrm{~mL}$ a nivel braquial. A cada muestra se le realizaron 
cuatro pruebas previamente estandarizadas para el diagnóstico de mansonela (Knott, gota gruesa, prueba en fresco y conteo por $\mathrm{mL}$ ). Se identificó cada una de las muestras y de las placas examinadas y se volvieron a examinar en el laboratorio clínico del hospital de Inírida. El profesional que practicó el estudio de las muestras es un bacteriólogo de planta que tiene experiencia en el diagnóstico de parásitos sanguíneos y que realizó entrenamiento de las técnicas aplicadas en el estudio. A los pacientes que refirieron síntomas, se les realizaron exámenes médicos y de laboratorio (cuadro hemático, gota gruesa, BK en esputo, etc.) para descartar patologías de la zona (paludismo, TBC, IRA, etc.).

Las pruebas de laboratorio se realizaron en cada una de las comunidades indígenas visitadas donde, mediante el muestreo aleatorio por familias (casas), se tomaron $4 \mathrm{~mL}$ de sangre venosa recolectada en tubo tipo venojet con EDTA como anticoagulante a cada uno de los integrantes de la vivienda. Este muestreo se realizó en la mayoría de las comunidades en horas de la mañana, excepto en la comunidad de Merey donde se realizó por la tarde.

Los pacientes seleccionados fueron mayores de 10 años, esto considerando que el período de incubación de esta enfermedad es largo, aunque hubo un paciente de 9 años positivo.

Cuando no fue posible el procesamiento de las muestras de sangre inmediatamente, se guardaron bien tapadas, a temperatura ambiente, máximo por 12 horas, tiempo en el cual se procesaban y el resto de muestra se guardaba en refrigeración.

Como una anotación importante, se observó que el hallazgo de las microfilarias en el método en fresco es mucho más fácil mientras se encuentren vivas, pues, su movimiento llama la atención cuando se recorre la placa en 100 aumentos; también es importante mencionar que ese movimiento se pierde al cabo de las 72 horas de recolección de la muestra.

Con cada una de las muestras tomadas, se desarrollaron las siguientes técnicas: gota gruesa, técnica de concentración de Knott, examen en fresco entre lámina y laminilla $(1,2,8,9)$ y recuento de parásitos en $50 \mu \mathrm{L}$ de sangre.

\section{Gota gruesa}

Se realizó mediante la siguiente técnica; sobre un portaobjetos limpio, se colocan dos gotas de sangre de aproximadamente $50 \mu \mathrm{L}$ de volumen, separadas la una de la otra por $2 \mathrm{~cm}$, aproximadamente; con la esquina de otro porta-objetos, se procede a la realización de los rectángulos con cada una de las gotas de sangre, igual al que se efectúa para el diagnóstico de paludismo $(1,2)$.

Una vez se secan a temperatura ambiente, se fijan con metanol durante un segundo; se dejan secar y, luego, se deshemoglobinizan con azul de metileno deshemoglobinizante (el mismo usado en paludismo) durante un segundo. Se escurren las láminas y se procede a su coloración con el colorante de Field; en esta ocasión, se utilizó uno preparado comercialmente (ALBOR $®$ ), el cual funcionó bien con 10 gotas de colorante diluidas en $3 \mathrm{~mL}$ de agua destilada; se mezcla y se colorean las placas por 10 min, tiempo en el cual se lavan con agua corriente, teniendo la precaución de rociar el agua suavemente, para no desprender la película de la sangre; una vez coloreadas, se dejan secar muy bien y se observan bajo inmersión.

\section{Técnica de concentración de Knott}

A un tubo graduado de centrífuga, se agregan $9 \mathrm{~mL}$ de formol al $10 \%$ (en agua destilada). Luego, se adiciona un $\mathrm{cm}^{3}$ de sangre y se completa hasta la marca 10; se mezcla bien por inversión, y se centrifuga por 10 minutos a 2500 rpm; se descarta el sobrenadante y del sedimento se hacen las preparaciones entre lámina y laminilla; con este mismo sedimento, se realizan las placas que posteriormente se van a usar para la identificación de especies una vez se hayan coloreado con Giemsa $(8,9)$.

\section{Examen en fresco y el recuento en $50 \mu \mathrm{L}$}

Se realizaron en la misma preparación, tomando con una pipeta automática $50 \mu \mathrm{L}$ de la sangre total previamente homogenizada y trasladándolos a un portaobjetos muy limpio; sobre la muestra se coloca una laminilla (se prefirieron 
las grandes, rectangulares) y se recorre toda la preparación en 10X (100 aumentos); el movimiento rápido de las microfilarias permite su localización rápida. Una vez se ha informado el examen en fresco como positivo, se procede con el recuento de los hemoparásitos presentes en toda la preparación y el resultado se multiplica por 20 que es el factor que permite convertir ese recuento en $50 \mu \mathrm{L}$ a $1000 \mu \mathrm{L}$ (equivalentes a un $\mathrm{cm}^{3}$ ).

El recuento de microfilarias por $\mathrm{cm}^{3}$ de sangre requiere tradicionalmente de dispositivos tales como cámaras que no están disponibles en el mercado colombiano. Otros procedimientos, como el recuento con base en el número de leucocitos por el método de gota gruesa, se pueden utilizar pero con el inconveniente de que en los pacientes con filariasis, no es tan exacto estandarizar el número de leucocitos por $\mathrm{mm}^{3}$ de sangre utilizando una constante, pues, las variaciones son notorias a diferencia de los recuentos leucocitarios de pacientes con paludismo. Además, baja su sensibilidad por usar menor cantidad de muestra.

Como un método práctico y rápido y con buen porcentaje de sensibilidad y especificidad, se puede realizar el recuento usando $50 \mu \mathrm{L}$ de sangre total no coagulada (EDTA), dosificada con la ayuda de una pipeta automática sobre un portaobjetos limpio para cubrir, luego, la preparación con un cubreobjetos de $22 \times 44 \mathrm{~mm}$, de los usados en fluorescencia, ya que con los de $22 \times 22 \mathrm{~mm}$ las preparaciones resultan demasiado gruesas y se dificulta la observación microscópica.

Esta técnica que no solamente sirve para recuento sino como tamizaje de positividad o negatividad, tiene algunas ventajas sobre los demás métodos de concentración, donde la vitalidad del parásito se ha perdido por el uso de colorantes como Giemsa o Wright $(8,9)$ o por formol. Algunas de éstas son:

1. Observando la preparación de la sangre fresca y con el objetivo de $10 \times$ (100 aumentos), se recorren todos los campos - de la forma usada para la lectura de las baciloscopias- en búsqueda de las microfilarias que llaman bastante la atención por sus continuos y bruscos movimientos.

2. Algunos micelios septados y continuos microsifonados al igual que las macroconidias en forma de clava de algunos mohos contaminantes pueden presentar similitudes morfológicas con las microfilarias cuando se emplean coloraciones como Giemsa o Wright; por ello es tan útil realizar la identificación exacta por su movimiento.

3. Paralelo al tamizaje, se puede realizar una estimación del número de microfilarias por $\mathrm{mm}^{3}$ de sangre, multiplicando por 20 el número de parásitos contados en la preparación, para obtener los resultados por cada por $\mathrm{cm}^{3}$ de sangre.

Lo anterior indica que la anterior metodología tiene una sensibilidad de 20 microfilarias por $\mathrm{cm}^{3}$ de sangre y que ante una prueba de concentración positiva como la de Knott u otras, y un resultado negativo por el método de conteo en $50 \mu \mathrm{L}$ de sangre fresca, se debe informar como menor de 20 parásitos por $\mathrm{cm}^{3}$ de sangre.

\section{Resultados}

En el estudio se encontraron los siguientes datos: la actividad predominante fue la de la agricultura que la realiza un grupo lingüístico mayoritariamente Curripaco donde el número de hombres y mujeres examinados fue similar. Los síntomas referidos durante el estudio en las personas examinadas fueron cefalea, fiebre, dolor de cuello, decaimiento y escalofríos. El diagnóstico final de las personas examinados fue: sanos, $58 \%$; pterigios, $32 \%$; embarazo, $2,8 \%$; rinofaringitis, $1,7 \%$, y otros, $5,5 \%$.

Los resultados de laboratorio mostraron una positividad para microfilariasis de $57,5 \%$ en la prueba de Knott, $42,5 \%$ en el examen de gota gruesa, $42 \%$ en el examen en fresco y $61,3 \%$ en el conteo por $\mathrm{cm}^{3}$. Al relacionar los positivos a la prueba de Knott por la sintomatología referida, se pudo encontrar una razón de prevalencia que mostró: ser agricultor y positivo para mansonelosis de una razón de prevalencia de 1,46 que fue estadísticamente significativa. 
Los síntomas, como la fiebre, presentaron una razón de prevalencia de 1,23 , estadísticamente significativa. La cefalea, el dolor de cuello, el escalofrío y el decaimiento dieron una razón de prevalencia de $1,47,1,55,1,35$ y 1,55 , respectivamente siendo todas estas razones estadísticamente significativas y relacionan estos síntomas con la presencia de enfermedad. No fue posible relacionar hallazgos físicos con la presencia de mansonela ya que a las personas examinadas no se les encontraron signos físicos relacionados con la enfermedad.

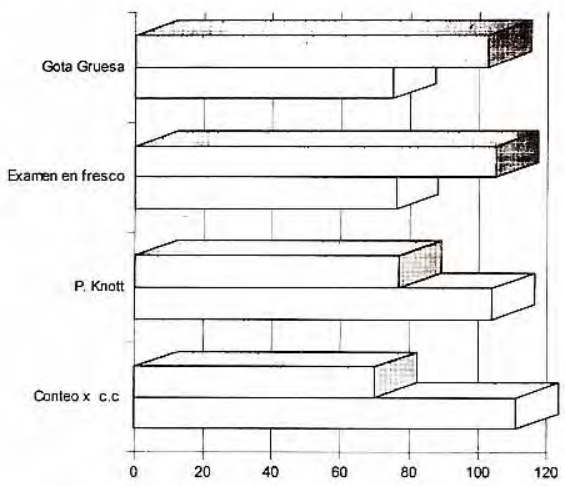

negativo $\square$ Positivo

Figura 1. Resultados de las pruebas de laboratorio. Comparación de pruebas de diagnóstico de mansonelosis.

Las comunidades evaluadas presentaron condiciones sociales, culturales, económicas, de vivienda y de saneamiento básico similares, presentando todas ellas como base de su economía la agricultura en especial la recolección de la fibra de chiqui-chiqui (cuadro 1).

Cuadro 1. Resultados de laboratorio de las muestras tomadas.

\begin{tabular}{lrcccc}
\hline $\begin{array}{l}\text { Prueba de } \\
\text { Laboratorio }\end{array}$ & Positiva $\%$ & Negativa & $\%$ & Total \\
\hline Examen en fresco & 76 & 42,0 & 105 & 58,0 & 181 \\
Prueba de Knott & 104 & 57,5 & 77 & 42,5 & 181 \\
Gota gruesa & 75 & 42,5 & 103 & 57,9 & 181 \\
Conteo por $\mathrm{cm}^{3}$ & 111 & 61,3 & 70 & 38,7 & 181 \\
\hline
\end{tabular}

Se puede apreciar cómo los casos positivos en las muestras en fresco fue de $76(42 \%)$, la prueba de Knott dio positividad en 104 (57,5\%) muestras y la prueba de gota gruesa mostró positividad en $75(42,1 \%)$.
Los resultados de laboratorio mostraron la presencia de microfilarias en las muestras procesadas, en los exámenes detallados para la identificación de las especies de filarias, realizados en primera instancia por el bacteriólogo de planta en el laboratorio del Hospital Inírida. Se detecto Mansonella ozzardi en las pruebas.

Para la zona del río Atabapo, en las comunidades examinadas, teniendo en cuenta la positividad de la prueba de Knott encontramos una proporción de prevalencia de $57,5 \%$ de los pacientes evaluados de acuerdo con la muestra calculada; si asumimos esta misma prevalencia para la población de la zona, encontramos que la enfermedad presenta una alta positividad para M. ozzardi.

En las pruebas de laboratorio, se realizó un recuento en la lectura de la prueba en fresco y se encontraron diferentes números de mansonela en las muestras, que varió de 1 a 233.

En la prueba cuantitativa (conteo de filarias por $\mathrm{cm}^{3}$ ), el número de parásitos varió de 0 a 4.660, en 181 muestras examinadas. Por este método, se logró detectar un número total de 111 pacientes positivos, mayor que el establecido en la prueba de Knott.

La cuantificación de filaria por $\mathrm{cm}^{3}$ varió de 20 parásitos a 4.660 por $\mathrm{cm}^{3}$. 98 de los pacientes estudiados tenían entre 20 y 200 parásitos por $\mathrm{cm}^{3}, 8$ presentaban entre 201 y 400 y 5 por encima de los 400 .

\section{Comparación de las pruebas de laboratorio}

Tomando los resultados de los exámenes de laboratorio, se pueden hacer los siguientes cálculos para establecer cual de las pruebas diagnósticas realizadas para las filarias es más sensible y específica, escogiendo como prueba de oro el examen mediante la técnica de Knott.

\begin{tabular}{|c|c|c|c|c|}
\hline & \multicolumn{2}{|c|}{ Knott } & \multirow[b]{2}{*}{ Total } \\
\hline & & + & - & \\
\hline & + & 71 & 5 & 76 \\
\hline Fresco & - & 33 & 72 & 105 \\
\hline \multicolumn{2}{|c|}{ Total } & 104 & $\pi 7$ & \\
\hline
\end{tabular}


Relacionando la prueba de Knott con el examen en fresco, encontramos los siguientes resultados:

S: $71 / 104=68,2 \%$

E: $72 / 77=93,5 \%$

VPP: $71 / 76=93,4 \%$

VPN: $72 / 105=68,5 \%$

Relacionando la prueba de Knott con la gota gruesa encontramos:

\begin{tabular}{ll|cc|c} 
& & \multicolumn{2}{|c|}{ Knott } & \multicolumn{1}{|c}{ Total } \\
\hline \multirow{2}{*}{ Gota gruesa } & + & 72 & 5 & 77 \\
& - & 33 & 72 & 104 \\
\hline & & 105 & 76 & 181
\end{tabular}

S: $72 / 105=68,5 \%$

E: $71 / 76=93,4 \%$

VPP: $72 / 77=93,5 \%$

VPN: $71 / 104=68,2 \%$

Al relacionar el conteo por centímetro cubico con la prueba de Knott, se dan los siguientes resultados:

\begin{tabular}{cc|rc|c} 
& & \multicolumn{2}{|c|}{ Knott } & Total \\
\hline \multirow{2}{*}{ Goteo por $\mathrm{cm}^{3}$} & + & 104 & 7 & 111 \\
& - & 0 & 70 & 70 \\
\hline & & 104 & 77 & 181
\end{tabular}

S: $104 / 104=100 \%$

E: $70 / 77=91,0 \%$

VPP: $104 / 111=93,6 \%$

VPN: $70 / 70=100 \%$

\section{Discusión}

\section{Pruebas de laboratorio en relación con la prueba de oro}

Tomando los exámenes de laboratorio realizados, se pueden hacer los siguientes cálculos para establecer cuál de las pruebas de laboratorio realizadas para el diagnóstico de filarias es más sensible y específica, escogiendo como prueba de oro el examen mediante la técnica de Knott.
Relacionando la prueba de Knott con el examen en fresco, encontramos los siguientes resultados. La sensibilidad de la prueba en fresco con relación a la de Knott es de $68,2 \%$ lo cual nos muestra que, a pesar de ser una prueba fácil de realizar en el campo, no tiene una sensibilidad tan alta como la prueba de Knott y deja de diagnosticar a casi $32 \%$ de los pacientes afectados por la enfermedad. Sin embargo, la prueba sí es altamente específica para las identificación del parásito cuando el paciente se encuentra afectado y clasificando por exclusión a las personas sintomáticas, teniéndolos como pacientes sanos. Con los valores predictivos se observa cómo el valor predictivo positivo es alto, con lo que se da una alta seguridad de no tener un número muy grande de falsos positivos; sin embargo, el valor predictivo negativo es bajo con lo cual el número de personas enfermas no fueron diagnosticadas adecuadamente mediante el examen en fresco de las muestras.

Relacionando la prueba de Knott con el examen de gota gruesa, observamos que la sensibilidad $(68,5 \%)$, especificidad $(93,4 \%)$ y los valores predictivos positivo $(93,5 \%)$ y negativo $(68,2 \%)$ son muy similares a los que observamos entre la prueba de Knott y el examen en fresco y su variación es muy escasa. Al igual que la prueba anterior, el examen de gota gruesa es poco sensible para la detección de los pacientes enfermos, altamente específico en determinar si están sanos, descartando los falsos positivos, pero presentando un porcentaje alto falsos negativos.

Al realizar el cruce de la información entre la prueba de Knott y los resultados positivos del examen de conteo por $\mathrm{cm}^{3}$ se puede apreciar que este sistema para el diagnóstico de mansonelas fue altamente sensible $(100 \%)$, específico $(91 \%)$ y de gran valor predictivo positivo $(93,6 \%)$ y negativo $(100 \%)$ en relación con la prueba de Knott. El examen de conteo identifica a los pacientes positivos para el parásito casi en igual condición que la prueba de Knott, escogida como la prueba patrón para el diagnóstico de microfilarias en el presente estudio. Por la facilidad en el procedimiento e identificación de las microfilarias, la capacidad de cuan- 
tificar los parásitos y los resultados encontrados, este examen de conteo por $\mathrm{cm}^{3}$ se convierte en una alternativa para el diagnóstico de filarias del tipo mansonela.

\section{Conclusiones}

Después de analizar los diferentes aspectos de la investigación y la comparación de los métodos diagnósticos en la prevalencia de filaria en comunidades del río Atabapo, departamento de Guainía, en 1996, se pudo llegar a las siguientes conclusiones:

1. La prevalencia de filaria en la zona estudiada del río Atabapo fue de $57,5 \%$ en el grupo de personas examinadas, donde se puede establecer en una primera instancia que son del tipo $M$. ozzardi; teniendo en cuenta que la muestra es representativa para la población de toda esta zona, podemos afirmar que la filaria presenta una alta prevalencia en las personas que viven en esta región. Si se tienen en cuenta los estudios anteriores como el de W. Kozek en 1982, donde se encontró una prevalencia de $25,8 \%$ para esta mansonelosis, se puede concluir que la enfermedad ha presentado un aumento considerable en la zona estudiada.

2. Se utilizaron para el diagnóstico de filariasis cuatro técnicas de laboratorio donde al prueba de Knott se tomó como la prueba de oro. Al relacionar las diferentes pruebas utilizadas en el estudio con la prueba de oro, se pudo establecer que el examen más fiable fue el de conteo por $\mathrm{cm}^{3}$ ya que esta técnica mostró la mayor sensibilidad, especificidad, valor predictivo positivo y valor predictivo negativo, en relación con el examen en fresco y la gota gruesa.

3. La mansonelosis dada por M. ozzardi en el área del río Atabapo se convierte en la enfermedad transmitida por vectores de mayor prevalencia en la zona y afecta a un gran número de personas de forma variable y por determinar.

\section{Recomendaciones}

1. Se hace necesario continuar con el estudio de mansonelosis en la zona del río Atabapo y en otras áreas del Guainía, debido a la alta prevalencia de esta parasitosis.

2. Para establecer programas de control, es necesario establecer los posibles vectores de $M$. ozzardi encontrados en la presente investigación mediante un estudio entomológico en la zona del río Atabapo.

3. Iniciar un plan de tratamiento a las personas afectadas por $M$. ozzardi, donde se estudie la efectividad, la seguridad y la disminución de la presencia del parásito en los pacientes, utilizando los medicamentos recomendados por la literatura. Se podría comparar la eficacia de varios medicamentos como la ivermectina, el levamizol y el albendazol.

4. Estudiar los hábitos de los vectores mediante el estudio de la periodicidad de las microfilarias en sangre, para así poder llegar a establecer si en esta zona $M$. ozzardi presenta algún ciclo o realmente no tiene ninguno como lo menciona la literatura médica mundial.

5. Aplicar como prueba de laboratorio de campo para el diagnóstico de microfilarias en sangre, la técnica de conteo por $\mathrm{cm}^{3}$ que mostró una sensibilidad y especificidad similar a la prueba de oro (técnica de Knott).

\section{Referencias}

1. Botero D, Restrepo M. Parasitología humana. Medellín: Ediciones Corporación para Investigaciones Biológicas; 1987:134-144.

2. Beaver PC. Clínica y complicaciones de las parasitosis. 1986:383-01.

3. Muñoz P. Simúlidos del río Chisaca (tesis). Bogotá: Universidad Nacional de Colombia, Facultad de Ciencias, 1990.

4. Manual de entomología médica para investigadores de América Latina. Cali: CIDEIM. 
5 Kozek W, Palma G, Colb. Filariasis en Colombia y Distribución de Mansonella ozzardi y Mansonella perstans en la comisaría del Guainía. Acta Médica 1985;16(3-4):137-42.

6. Marinkelle CJ, German E. Mansonelliasis in the Comisaria del Vaupés of Colombia. Trop Geogr Med 1970;22:101-11.

7. Perfil epidemiológico del departamento del Guainía, 1996; Secretaria de Salud del Guainía; 1996.

8 Badwin M, Slaten D, Maloni J. Comparación de dos técnicas de Knott y filtro de nucleopore en el diagnóstico de filariasis por $W$. bancrofti. Boletín Oficina Sanit Panam 1982;9(1):13-7.

9. Klanger S, Whitworth JA. Laboratory diagnosis of filariasis. Clin Lab Med 11(4):977-1010.

10. Kozek WJ, Palma G, Colb. Filariasis en Colombia: prevalencia de Mansonella ozzardi en el departamento del Meta, intendencia del Casanare y comisaría del Vichada. Acta Médica 1986;17(1):40-2.

11. Botero D, Restrepo M. Parasitología humana. Medellín: Ediciones Corporación para Investigaciones Biológicas; 1987:134-44.

12. Acosta BE. Caso humano de Wuchereria bancrofti, datos experimentales con Culex fatigans y Aedes aegypti. Universitas 1951;1:71-97.

13. Little MD, Assis-Masri G. A case of ocular onchocercosis in Colombia. Trans Roy Soc Trop Med Hyg 1995;59(6):717.

14. Ewert A, Corredor A, Lightner L. Onchocersiasis focus in Colombia: follow-up study after 12 years. Am J Trop Med Hyg 1979;28(3):486-90.

15. Little MD, D'Alessandro A. Onchocerciasis in Colombia. Am J Trop Med Hyg 1970;19(5):831-6.
16. Trapido H, D'Alessandro A, Little MD. Onchocercosis in Colombia. Historical background and ecologic observations. Am J Trop Med Hyg 1971; 20(2):104-89.

17. Barreto P, Trapido H. Onchocerciasis in Colombia: entomologic finding in the first observed focus. Am J Trop Med Hyg 1970;19(5):837-41.

18. Palma G, Travi B ¿Oncocercosis en Colombia? Una revaluación del foco de López de Micay. Biomédica 1995; 15:131-6.

19. Marinkelle CJ, German E. Mansonelliasis in the comisaria del Vaupés of Colombia. Trop Geogr Med 1970;22:101-11.

20. Comité Nacional de Oncocercosis. Plan nacional de erradicación, 1995.

21. Chiquito M, Cueto V. Informes de bacteriología de la comisión multidiciplinaria al río Guanía, 1995.

22. Muñoz de Hoyos P. Simúlidos del río Chisacá. (tesis). Bogotá: Universidad Nacional de Colombia, Facultad de Ciencias, 1990.

23. Godman y Gilman. Las bases farmacológicas de la terapéutica. Séptima edición. Editorial Médica Panamericana, 1980.

24. Beaver PC. Clínica y complicaciones de las parasitosis. 1986;23:383-01.

25. Padilla JC. Prevalencia de filarias en el departamento del Chocó, 1993. Informe preliminar. SEM, regional Chocó.

26. Morales M, Almeida M. Mansonella ozzardi entre indios Ticunas do estado do Amazonas, Brasil. Boletín Oficina Sanit Panam 1978;85(1):16-23.

27. Manual de entomología médica para investigadores de América Latina. Cali: CIDEIM. 